\title{
An Energy-Resolved Optical Non-invasive Device Detects Essential Electrolyte Balance in Humans at Point-of-Care
}

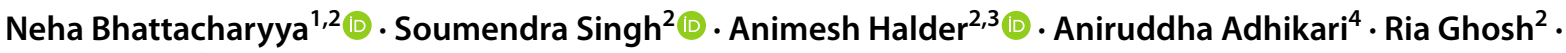 \\ Deep Shikha ${ }^{2} \cdot$ Santanu Kumar Tripathi ${ }^{5} \cdot$ Asim Kumar Mallick ${ }^{6} \cdot$ Pulak Mondal $^{1} \cdot$ Samir Kumar Pal ${ }^{2,4}$ (1)
}

Received: 1 November 2020 / Accepted: 21 January 2021 / Published online: 11 February 2021

(c) Indian National Academy of Engineering 2021

\begin{abstract}
Regular monitoring of electrolyte balance is essential for patients suffering from chronic kidney disease (CKD), particularly those undergoing dialysis. In the context of the recent COVID-19 pandemic, more severe forms of infection are observed in elderly individuals and patients having co-morbidities like CKD. The repeated blood tests for the monitoring of electrolyte balance predispose them not only to COVID-19 but also other to hospital-acquired infections (HAI). Therefore, a non-invasive method for easy detection of essential electrolyte $\left(\mathrm{K}^{+}\right.$and $\left.\mathrm{Na}^{+}\right)$levels is urgently needed. In this study, we developed an optical emission spectroscopy-based non-invasive device for simultaneous monitoring of salivary $\mathrm{Na}^{+}$and $\mathrm{K}^{+}$levels in a fast and reliable way. The device consisted of a closed spark chamber, micro-spectrometer, high voltage spark generator, electronic circuits, optical fiber, and an indigenously developed software based on the LabVIEW platform. The optical emission originating from the biological sample (i.e., saliva) due to recombination of ions energized by impingement of electrons returning from high voltage spark provides necessary information about the concentration of electrolytes. A small-scale clinical trial on 30 healthy human subjects shows the potential of the indigenously developed device in determining salivary $\mathrm{Na}^{+}$and $\mathrm{K}^{+}$ concentration. The low-cost, portable, point-of-care device requires only $2 \mathrm{~mL}$ of sample, and can simultaneously measure $1.0-190.0 \mathrm{mM} \mathrm{Na}^{+}$, and $1.0-270.9 \mathrm{mM} \mathrm{K}^{+}$. To our understanding, the present work will find its relevance in combating COVID-19 morbidities, along with regular CKD patient-care.
\end{abstract}

Keywords Non-invasive essential electrolyte measurement $\cdot$ Spectroscopic instrument $\cdot$ CKD management

Samir Kumar Pal

skpal@bose.res.in

1 Department of Radio Physics and Electronics, University of Calcutta, 92, Acharya Prafulla Chandra Rd, Machuabazar, Kolkata 700009, India

2 Technical Research Centre, S. N. Bose National Centre for Basic Sciences, Block JD, Sector III, Salt Lake, Kolkata 700106, India

3 Department of Applied Optics and Photonics, University of Calcutta, Block JD, Sector III, Salt Lake, Kolkata 700106, India

4 Department of Chemical, Biological and Macromolecular Sciences, S. N. Bose National Centre for Basic Sciences, Block JD, Sector III, Salt Lake, Kolkata 700106, India

5 Department of Clinical and Experimental Pharmacology, Calcutta School of Tropical Medicine, 108, Chittaranjan Avenue, Kolkata 700073, India

6 Department of Paediatric Medicine, Nil Ratan Sircar Medical College and Hospital, 138, AJC Bose Road, Sealdah, Raja Bazar, Kolkata 700014, India

\section{Introduction}

The SARS-CoV-2 virus which causes the COVID-19 infection has immensely impacted the world and caused a global pandemic with over 91 million infections and over 1.9 million fatalities till January 2021 (JHU 2021). Patients, especially those with kidney transplants, cardiovascular diseases, and diabetes are at high risk of COVID-19 infection. Moreover, patients undergoing hemodialysis have to visit medical centers frequently, thereby loses their ability to self-isolate and become prone to nosocomial infections (Ajaimy and Melamed 2020). While looking at the pathophysiology of COVID-19, many researchers are focusing attention on a specific protein, namely, Angiotensin-Converting Enzyme 2 (ACE 2) that aids the virus to infect the human cells. The spike protein on the surface of the SARS CoV-2 virus attaches to ACE 2 for their entry to human cells and replicates to spread infection. This becomes lethal for patients having chronic kidney diseases (CKD). ACE 2-inhibition 
by the virus also leads to vasoconstriction increasing blood pressure and causes hypernatremia leading to water retention in the body resulting in electrolyte imbalance in humans (Behl et al. (2020); Mizuiri and Ohashi 2015; Soler 2013). The relative expression of ACE and ACE 2 synergistically controls the electrolyte balance (Wakahara 2007; Pinheiro 2019; Rieder 1999). In CKD patients, disruption in the balance between intra-renal ACE and ACE 2 expression gives rise to high levels of angiotensin 2 , contributing to the progression of renal damage (Hamming 2007). Thus, necessary diagnostic information for the treatment of several diseases like CKD, acute cardiac arrhythmias hypertension, etc. is determined based on the analysis of the vital electrolyte levels, especially sodium $\left(\mathrm{Na}^{+}\right)$and potassium $\left(\mathrm{K}^{+}\right)$in the human body (Garcia 1992). In this context, Hyponatremia, another very common electrolyte disorder, especially in the elderly, is associated with significant morbidity, mortality, and disability in the context of the COVID-19 pandemic. Thus, easy access to the essential electrolyte monitoring (preferably at home) in a non-painful way is essential to control the comorbidity of CKD and elderly subjects in the context of the COVID-19 pandemic (Fraser and Blakeman 2016).

Despite being the most familiar analytes, very few techniques are available for the measurement of $\mathrm{Na}^{+}$and $\mathrm{K}^{+}$ in clinical chemistry laboratories. Ion-selective electrodes (ISEs), flame photometry, and atomic absorption spectrometry (AAS) are the conventional technologies for quantifying the essential ion levels in plasma or other biological fluids (Giavarina 2019; Rose 2014; Lee 2019; Delanghe 2019). Although ISEs, AAS, and flame photometry are used extensively, some limitations exist. High cost, immobility, utilization of various chemical reagents, use of dedicated electrodes for each electrolyte, the requirement of an expert operator, are a few to mention (Durst 1978; Delves 1987; Diwakar and Kulkarni 2012). These drawbacks make it difficult to use the aforementioned methodologies in point-ofcare diagnostics, which is vital for the current healthcare situation. Depending on the type of instrument, the sample is selected from urine, saliva, and whole blood. Extraction of blood by pricking needles is not only painful but also has the risk of excessive bleeding, infection, pain, loss of consciousness, and multiple hospital visits which may cause Hospital Acquired Infections (HAI) (Zungu et al. 2008). Thus, low-cost non-invasive techniques for the detection of electrolyte levels from other biological fluids, are preferred. A recent study from our group used a spark emission spectroscopy-based method for the detection of electrolyte levels from the human blood serum (Halder 2019). However, no detailed study using other body fluids was performed. Moreover, the method required a great volume of sample for detection which makes it a bit difficult for clinical diagnosis at point-of-care. In this study, we have successfully lowered the minimum detectable $\mathrm{Na}^{+}$level to $1 \mathrm{mM}$ from $100 \mathrm{mM}$. Installation cost and cost per sample test have been decreased to a substantial amount. Thus, the primary objective of the present work is to develop a non-invasive, portable device that would be able to detect the concentration of electrolyte contents in humans at point-of-care using the wash of oral cavity without a rigorous sample preparation procedure.

\section{Materials and Methods}

\section{Instrumental Setup}

Figure 1a schematically describes the detailed arrangement of the device, which includes a closed spark chamber (CSC), vaporizer, micro-spectrometer, high voltage control electronics, optical fibers, and an indigenously developed software. Figure 1b, c show the digital photographs of the prototype device. The CSC, a metal tube of $1.9 \mathrm{~cm}$ diameter, comprises two opposing copper electrodes (standard spark plug, Model- CPR8EA-9, NGK Spark Plug India Pvt. Ltd., India) having a $0.1 \mathrm{~cm}$ distance in between (Fig. 1a) for spark discharge (Staack 2008; Singh 2014; Hnatiuc et al. 2011; Sher et al. 1992; Shrivastava and Gupta 2011). Intending to reduce the manufacturing cost, we used commercially available copper electrodes having a very low corrosion rate instead of noble metal electrodes. The low corrosion rate ensures no possible interference with optical observations to occur. The CSC also hosts a multimode optical fiber (numerical aperture: 0.10; core: $10.5 \mathrm{~cm}$; Thor Labs, USA) placed at a right angle to the spark plug at a distance of $0.85 \mathrm{~cm}$ from the center to carry the optical information to the microspectrometer. The micro-spectrometer was developed using a MEMS-based chip from Hamamatsu Photonics, Japan (Model: C12880MA). The high voltage electronics include a transformer that supplies the required voltage to generate a spark. The vaporizer breaks the liquid sample into small aerosol droplets and pushes them to the CSC.

\section{Working Principle}

The working principle remained the same as our previous study (Halder 2019). The major modification in the setup i.e., closing the spark chamber resulted not only in the requirement of less amount of sample but also reduction of fluctuations. In brief, spark plugs in the CSC generate plasma utilizing high voltage sources and excite the vaporized samples (i.e., aqueous solutions of electrolytes, or biological fluids). The optical emission originating from each sample (i.e., optical plume, Fig. 2a) due to recombination of ions energized by impingement of electrons resulting from high voltage plasma discharge is collected by using the 


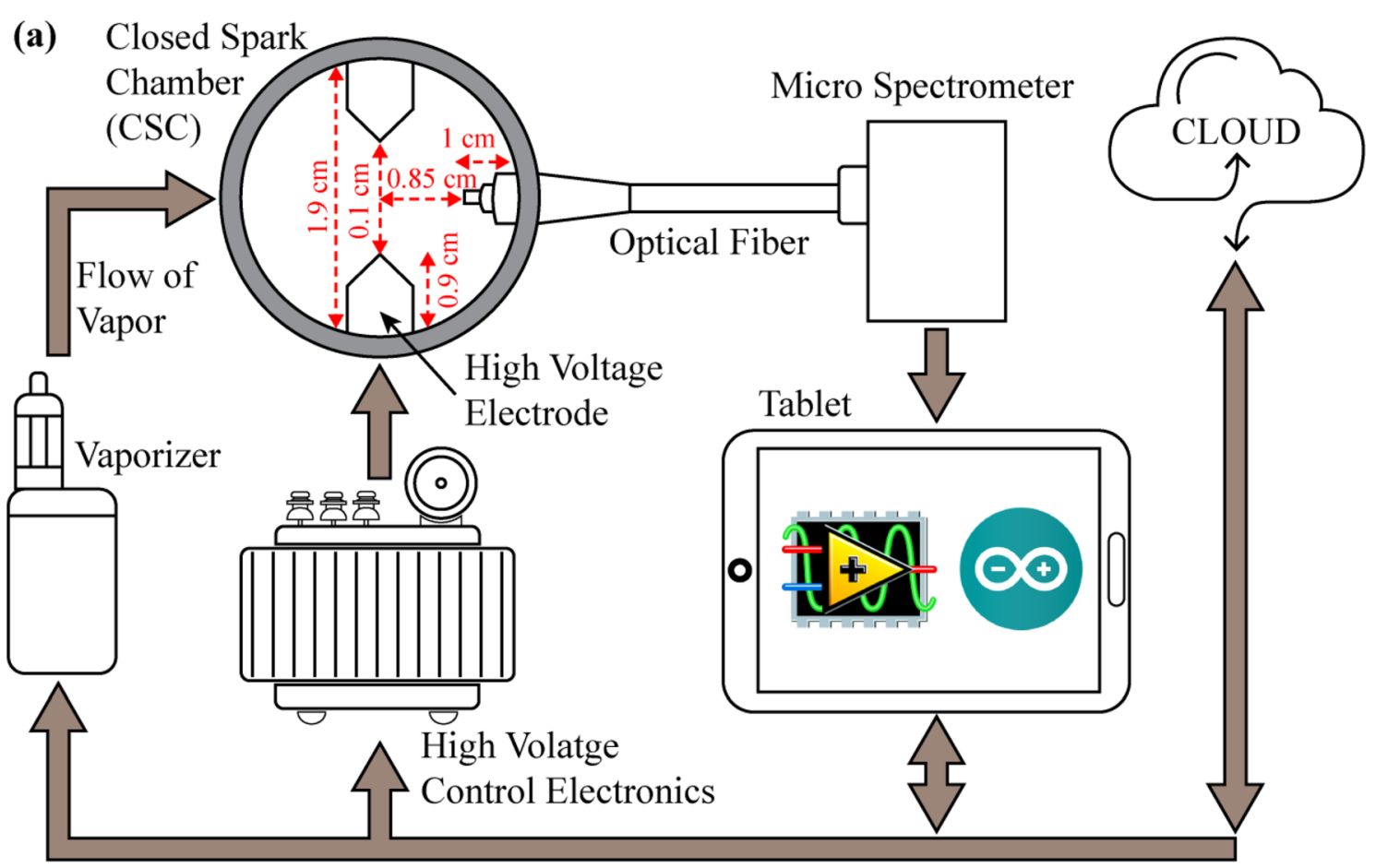

(b)

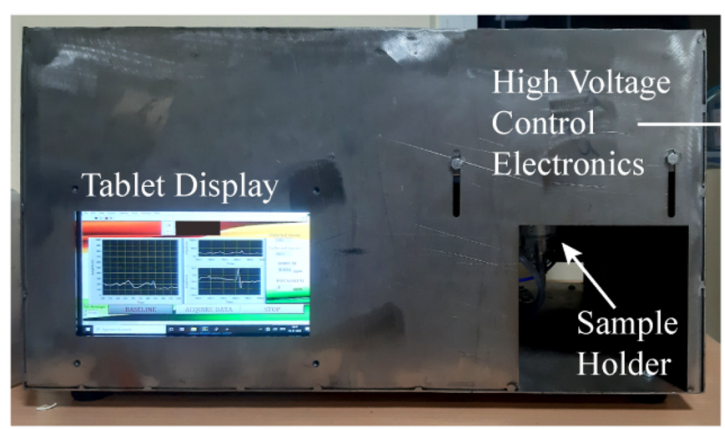

(c)

TOP VIEW

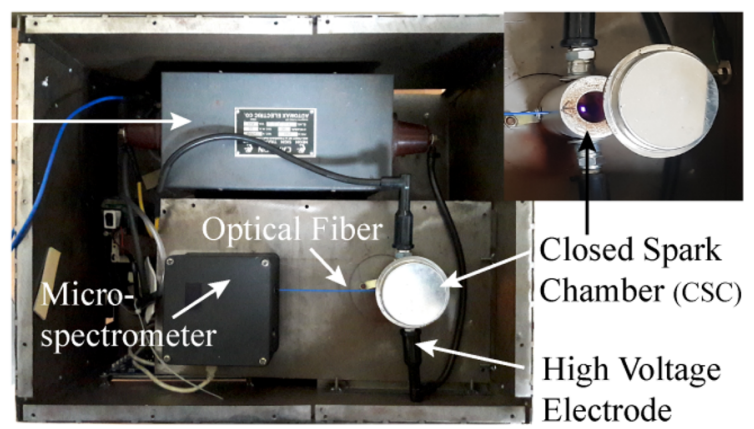

Fig. 1 a The schematic of the experimental arrangement for non- invasive detection of essential electrolytes in humans. b, c The digital photographs of the prototype device

optical fiber setup and detected by the micro-spectrometer. The control of the system including interfacing and IoT strategy is executed by an indigenously developed software in the LabVIEW platform (National Instruments, USA).

\section{Calibration of the Device}

For calibration of the instrument, different concentrations of sodium chloride ( $\mathrm{NaCl}$; Sigma, USA) and potassium chloride ( $\mathrm{KCl}$; Sigma, USA) solutions were prepared using Millipore water. The concentrations of $\mathrm{NaCl}$ used were 25,50 , $75,100,150$, and $200 \mathrm{mgL}^{-1}$. While for $\mathrm{KCl}$, the concentrations were $50,75,100,150$, and $200 \mathrm{mgL}^{-1}$. The chemicals were of the highest commercially available grade and used without further purification.
For measurement, a $2 \mathrm{~mL}$ solution was placed in the sterilized sample holder and subsequently measured using the principle stated in the previous section. Instrument index value (i.e., the emission intensity corresponding to the emission peaks of $\mathrm{Na}^{+}$, and $\mathrm{K}^{+}$ions) was obtained for each calibrating sample and finally correlated with the known concentrations to obtain the calibration equations which were included in the software (vide infra). In between two consecutive sets of measurements, the spark plugs were turned on for 25 min to clean the electrodes and avoid possible contamination among samples. 

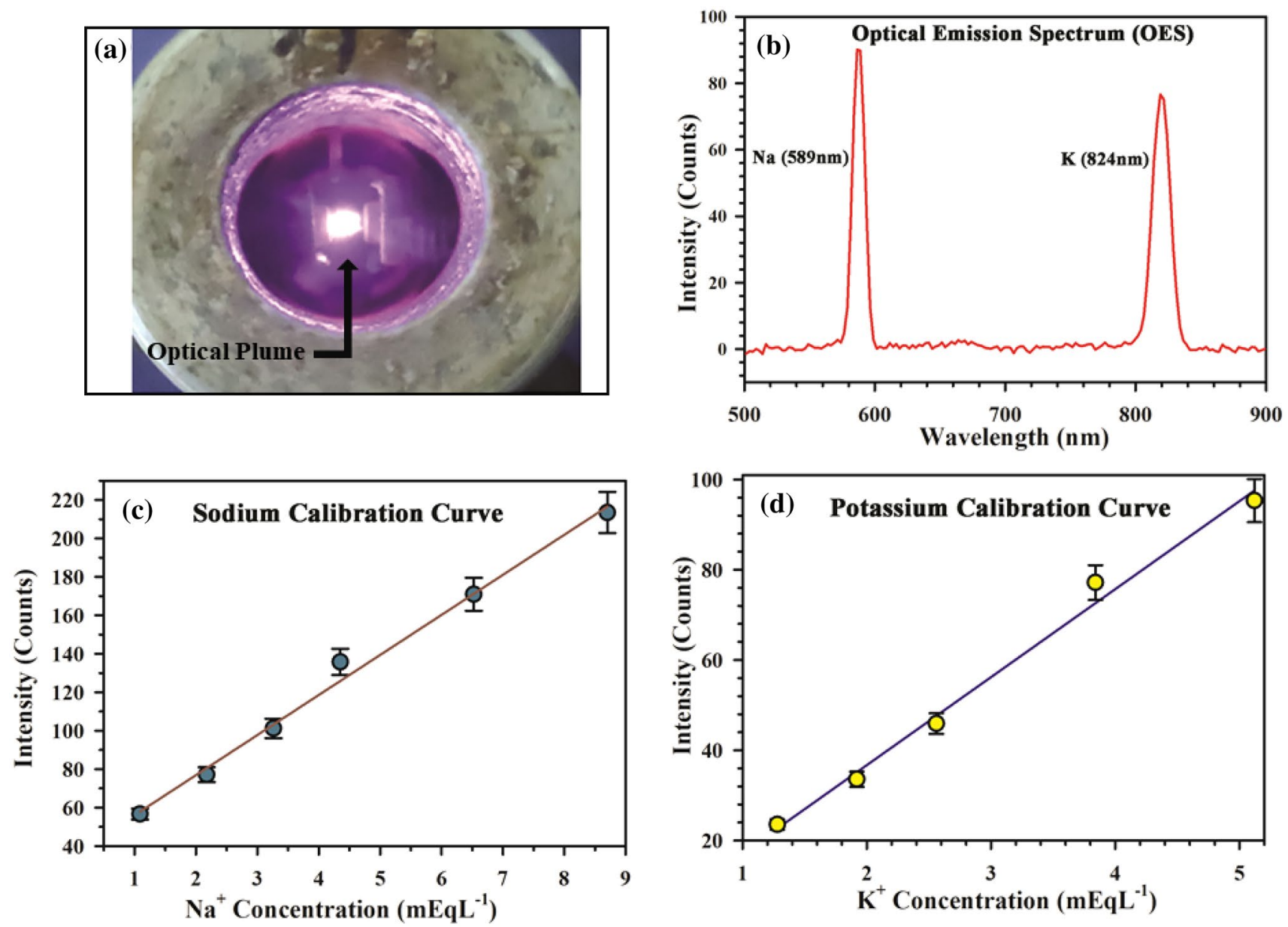

Fig. 2 a The high voltage electrodes generating the spark. b The spectrum where the individual peaks for $\mathrm{Na}^{+}$and $\mathrm{K}^{+}$can be seen. $\mathbf{c}, \mathbf{d} \mathrm{Show}$ the calibration curves obtained for $\mathrm{Na}^{+}$and $\mathrm{K}^{+}$with $\pm 5 \%$ error, respectively

\section{Validation of the Device using Biological Fluids}

\section{Study Population and Ethical Considerations}

The study was conducted following the guidelines approved by the Institutional Ethics Committee (Ref: CREC-STM/2018-AS27, dated 29.12.2018). All studies involving human subjects were performed according to the guidelines of the Declaration of Helsinki (Declaration 2014) and Indian Council for Medical Research (ICMR), Govt. of India.

As a proof-of-concept study, the sample size was estimated to be 30 . Written informed consent was obtained from the volunteers who agreed to participate in the study after understanding the particulars and consequences of the study. All data and information about the subjects are kept confidential and utilized only for this study.

\section{Sample Collection}

After preliminary mouthwash with regular water, $4 \mathrm{~mL}$ of deionized water was poured into the oral cavity of the subject. After one minute, the water mixed saliva i.e., the sample $(\sim 4.5 \mathrm{~mL})$ was collected. $1.5 \mathrm{~mL}$ of the collected sample was diluted with $0.5 \mathrm{~mL}$ of deionized water, placed into the sterilized sample holder, and subsequently measured using the method described in the earlier section. From the instrument index values, respective concentrations of the ions were obtained according to the calibration equations. Multiplying the obtained values with proper dilution factor provided actual levels of $\mathrm{Na}^{+}$and $\mathrm{K}^{+}$in the saliva. 


\section{Statistical Analysis}

Data are expressed as Mean \pm Standard Deviation (SD) unless otherwise stated. All statistical analyses including linear, and non-linear regression were performed using Origin Pro 8.5 (Origin Lab, USA). For reference interval calculations we used the parametric method considering the Gaussian distribution of the values. The difference between study results and reference standards was estimated using an unpaired $t$ test (GraphPad Prism 9.0, GraphPad Software, USA). $P<0.05$ were considered significant.

\section{Results and Discussion}

In the present study, we used the energy-resolved optical emission signatures associated with $\mathrm{Na}^{+}$and $\mathrm{K}^{+}$for the determination of their respective concentrations in the samples of interest. Figure $2 \mathrm{~b}$ shows the emission spectral pattern of the electrolytes. The observed peaks at 589 and $824 \mathrm{~nm}$ corresponds to $\mathrm{Na}^{+}$and $\mathrm{K}^{+}$, respectively (Slanger and Osterbrock 2000). As stated in the methods section, calibration curves were obtained using different concentrations of $\mathrm{Na}^{+}$and $\mathrm{K}^{+}$solutions. As evident from Fig. 2c, d, emission intensities were linearly dependent upon the electrolyte concentrations for both $\mathrm{Na}^{+}$and $\mathrm{K}^{+}$. Linear regression analysis leads to the following calibration equations with adj. $R^{2}$ of $0.999\left(\mathrm{Na}^{+}\right)$, and $0.988\left(\mathrm{~K}^{+}\right)$.

Intensity $\mathrm{Na}^{+}($counts $)=23.31+20.82$ Concentration $_{\mathrm{Na}^{+}}(\mathrm{mEq} / \mathrm{L})$

Intensity $_{\mathrm{K}^{+}}($counts $)=-2.29+19.5$ Concentration $_{\mathrm{K}^{+}}(\mathrm{mEq} / \mathrm{L})$

where, Intensity i.e., instrument index value refers to the optical emission intensities (counts) of the electrolytes at their respective peaks, and concentration refers to the concentration of the samples in the holder. The calibration equations served as standard curves and were incorporated into the software to calculate electrolyte concentrations of unknown samples from their emission intensities.

Next, we aimed to quantify $\mathrm{Na}^{+}$and $\mathrm{K}^{+}$concentrations from salivary samples. Salivary glands are non-excitable effector organs in which appreciable translocation of fluid and electrolytes occurs from the interior of the organism to the outside, usually in response to the neural stimulation (Schneyer et al. 1972). The salivary secretion does not regulate the body's electrolyte balance, rather functions as an indicator to it. It is even considered a better physiological marker of electrolyte imbalance than the serum or blood. Despite having huge potential as a disease marker, the salivary electrolyte levels received less attention in medical diagnosis due to the unavailability of standardized measurement techniques in clinical chemistry laboratories. The first hurdle in measuring electrolyte concentration from saliva
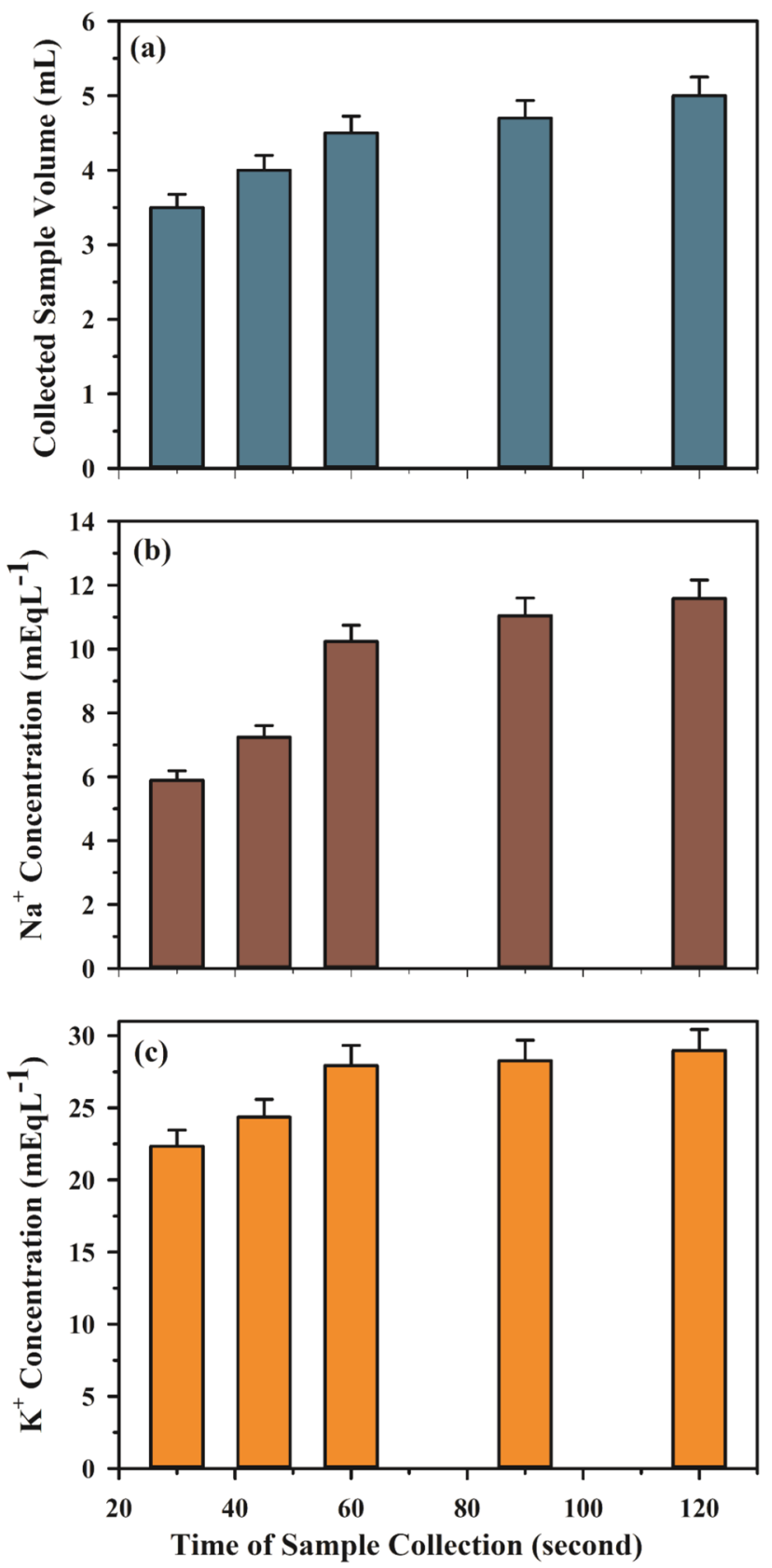

Fig. 3 a The volume of salivary secretion collected at different time intervals (60-120 s). b, c Respectively show the salivary $\mathrm{Na}^{+}$and $\mathrm{K}^{+}$ concentration during this period

was to standardize the sample collection protocol. Here, we used a non-stimulated method by washing the oral cavity with $4 \mathrm{~mL}$ of deionized water for $60 \mathrm{~s}$. The comprehensive wash of the oral cavity for $60 \mathrm{~s}$ is expected to overcome the physiological factors on which salivation depends. As showed in Fig. 3a, the volume of salivary secretion by this method reached a stable condition between 60 and $120 \mathrm{~s}$. The salivary $\mathrm{Na}^{+}$or $\mathrm{K}^{+}$concentration also remained nearly constant during this period (Fig. 3b, c). Furthermore, $<60 \mathrm{~s}$ 

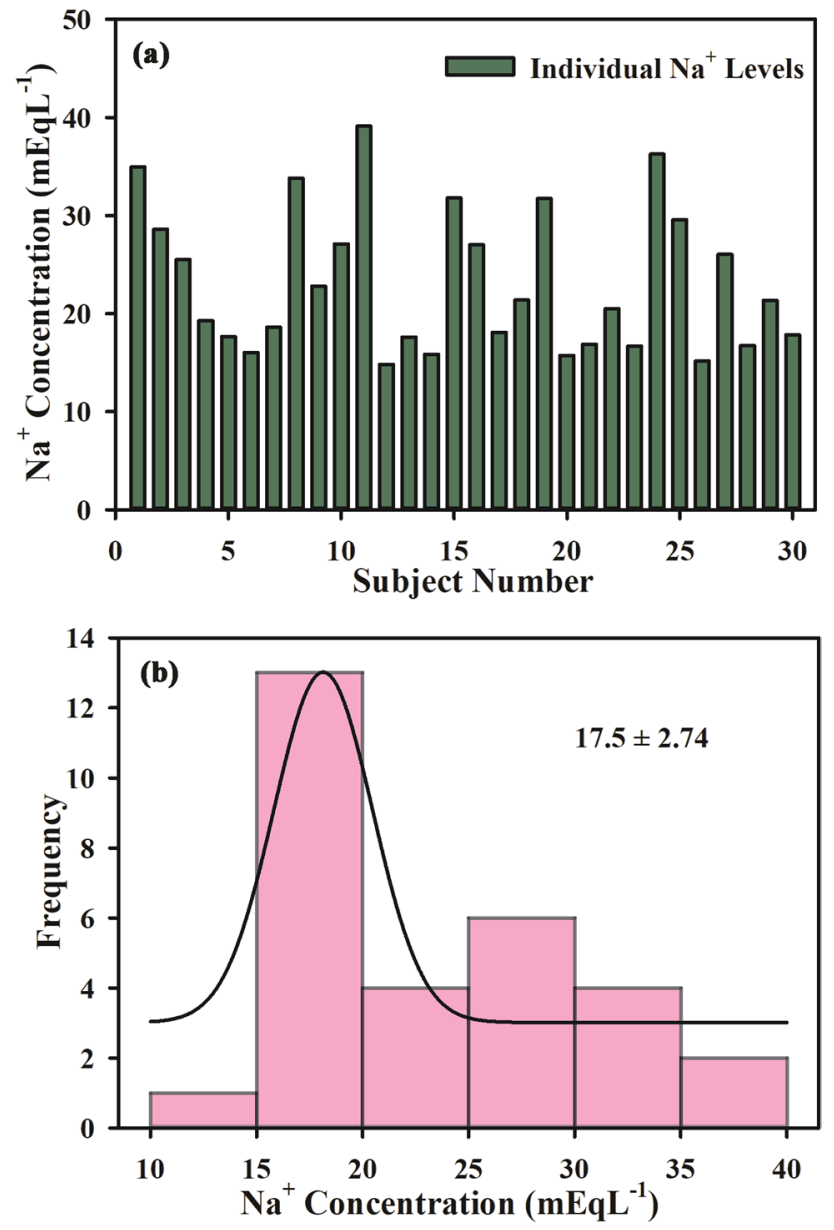

Fig. 4 a The individual $\mathrm{Na}^{+}$levels of 30 subjects. b The statistical analysis of the same

is too little to monitor for the patients suffering from acute illness. Therefore, the washing time was set at $60 \mathrm{~s}$.

Figure 4a shows the salivary $\mathrm{Na}^{+}$values of each patient obtained using our device. As all the volunteers were healthy in terms of electrolyte balance, we plotted a frequency distribution curve for salivary $\mathrm{Na}^{+}$concentration (Fig. 4b) to estimate the reference range for the study population. The observed frequency distribution curve for salivary $\mathrm{Na}^{+}$levels showed a bell-shaped pattern (i.e., Gaussian distribution) indicating normal distribution, one of the major criteria for reference range determination. The central tendency (i.e., mean) was found to be $17.5 \mathrm{mEqL}^{-1}$, with a $2 \mathrm{SD}$ of $2.7 \mathrm{mEqL}^{-1}$ (i.e., full-width-half-maxima of the frequency distribution plot). As per definition, the reference range for a given population for a given parameter (i.e., analyte or measurand) ranges from Mean $+2 \mathrm{SD}$ to Mean-2SD. It is expected that $95 \%$ of the individuals if tested, will have their analyte values within this range. Thus, in our case, the reference range for salivary $\mathrm{Na}^{+}$was found to be $14.8 \mathrm{mEqL}^{-1}$ (lower limit of variation) to $20.2 \mathrm{mEqL}^{-1}$ (upper limit of variation).
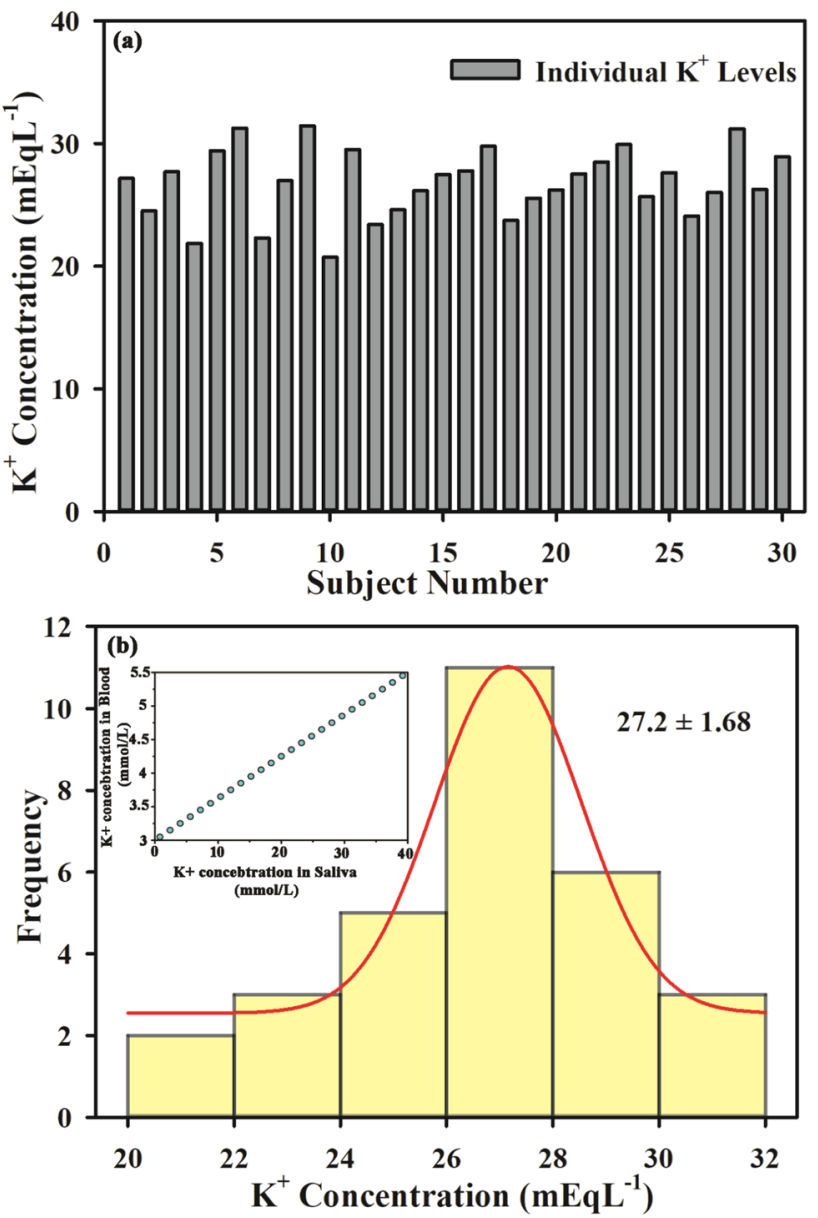

Fig. 5 a The individual $\mathrm{K}^{+}$levels of 30 subjects. b The statistical analysis of the same. The inset shows the correlation of the $\mathrm{K}^{+}$levels from saliva and blood plasma as obtained from the reported data (Horiba 2017)

The calculated limits of variation were in good agreement with the published limits of salivary $\mathrm{Na}^{+}$concentration $\left(26.4 \pm 11.8 \mathrm{mEqL}^{-1}\right)$. It is worth mentioning here that, if the population is healthy and the instrument provides accurate measurements, a frequency distribution curve, would tend to have the same general shape if the number of population is increased, or if the groupings were varied to some small degree (White 1955; Chiles et al. 1996). Therefore, the observed statistically insignificant difference between the frequency distribution curve obtained from the developed device and previously published values, in turn, indicates the efficacy of the device in clinical settings.

Figure 5a shows the salivary $\mathrm{K}^{+}$levels of the 30 healthy volunteers who participated in the study. Figure $5 \mathrm{~b}$ shows the frequency distribution curve. Similar to $\mathrm{Na}^{+}$, the frequency distribution curve for salivary $\mathrm{K}^{+}$levels were found to be bell-shaped. The central tendency was $27.2 \mathrm{mEqL}^{-1}$, with a $2 \mathrm{SD}$ of $2.7 \mathrm{mEqL}^{-1}$. The reference range for salivary $\mathrm{K}^{+}$was calculated to be $25.5 \mathrm{mEqL}^{-1}$ (lower limit of 
variation) to $28.9 \mathrm{mEqL}^{-1}$ (upper limit of variation). The observed limits of variation showed no significant difference from the previously published reference range which is $19.7 \pm 3.9 \mathrm{mEqL}^{-1}$ Therefore, the salivary $\mathrm{K}^{+}$concentrations measured by the developed device efficiently mimicked corresponding clinical values, indicating the efficiency of the instrument in clinical settings.

Previous studies have revealed that in both hypotonic and isotonic human saliva the concentration of $\mathrm{Na}^{+}$ions is less compared to serum, while $\mathrm{K}^{+}$concentration is frequently higher than the serum level of this ion. In our study too, we observed similar results. The salivary $\mathrm{Na}^{+}$level found in our study is $\sim 8$ times lower than that found in serum. Similarly, the salivary $\mathrm{K}+$ concentration in this study is $\sim 7$ times higher compared to serum. The higher concentration of $\mathrm{Na}^{+}$ observed in the blood could be due to the higher volume and surface area of blood (Wankasi 2019). Moreover, blood is an extracellular fluid from where a lot of nutrients and electrolytes are cleared out from the body or transported to other cells, tissues, and body fluids for systemic sustenance (Wankasi 2019). On the other hand the high concentration of $\mathrm{K}^{+}$in saliva is due to the filtration of potassium into the saliva and the active release to the saliva by nerves stimulation as reported by Carlos et al. (Labat 2018). Also, $\mathrm{Na}^{+}$ions are actively reabsorbed from all the salivary ducts and $\mathrm{K}^{+}$ ions are actively secreted in exchange for the $\mathrm{Na}^{+}$(Wankasi 2019). Therefore, the $\mathrm{Na}^{+}$ion concentration of the saliva becomes greatly reduced, while the $\mathrm{K}^{+}$ion concentration becomes increased. Figure $5 \mathrm{~b}$-inset shows a sample correlation between serum and salivary concentration of $\mathrm{K}^{+}$ (Horiba 2017). However, we found no authentic report that established a similar correlation between serum and salivary $\mathrm{Na}^{+}$concentration. Therefore, a detailed large-scale systemic clinical study is required to find the exact correlation between serum and salivary electrolytes, which we are planning to do in the future.

It is worth mentioning here, the salivary electrolyte concentration is very much dependent on circadian rhythm. It also varies greatly depending upon the time of daily meal intake. We have measured the salivary electrolyte concentrations at different times of the day and observed the variation as depicted in Fig. 6. However, for maintaining uniformity, we collected all the samples used for the determination of the reference range at fasting conditions. This kind of strategy is often used while measuring certain blood parameters to avoid diurnal variations.

This is a very preliminary proof of concept study, the sole aim of which was to test whether the newly developed device can measure salivary $\mathrm{Na}^{+}$and $\mathrm{K}^{+}$concentration from the mouth wash as an alternative to the conventional invasive electrolyte detection techniques. Previously, we also proposed a device with the same working principle for the detection of analytes from blood samples. Here, the

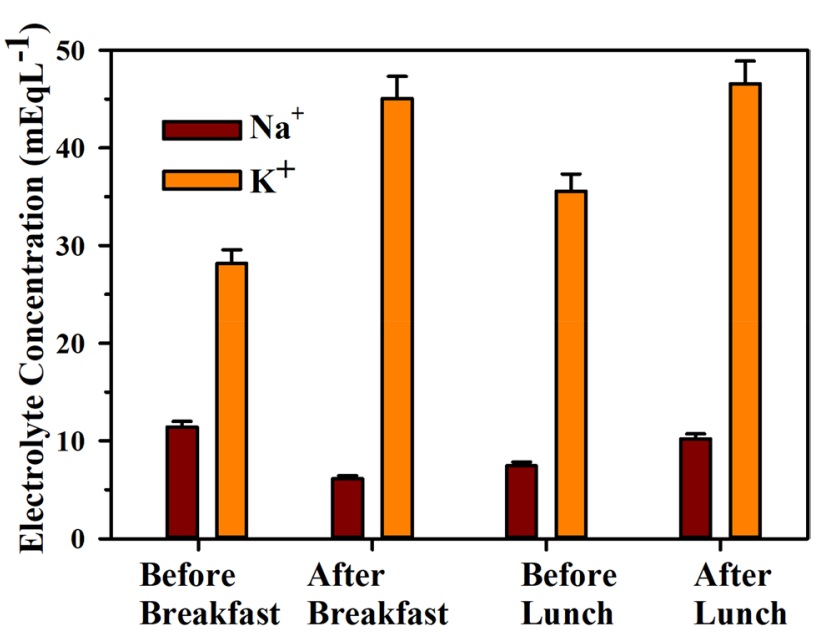

Fig. 6 Measured salivary electrolyte concentrations at different times of the day

installation and maintenance cost, and amount of sample required for testing have been reduced to a remarkable extent compared to our previously proposed device. Due to covered CSC, and more integrated systems the proposed device causes no known medical hazard since no chemical or biomedical waste is exhausted (unlike our previous design) to the surroundings. Moreover, it helped to lower the minimum detection limit for $\mathrm{Na}^{+}$to $1 \mathrm{mM}$, which adds to its clinical significance. Table 1 depicts a detailed comparative analysis of our proposed device with other standard devices and our previous design.

There are some limitations of the study, which lay the foundation stone for future investigations. First of all, we restricted our study to only normal volunteers since we did not have ethical permission to work on the patients, particularly the COVID-19 infected ones. It is of particular concern whether cough and cold could affect our measurements or salivary electrolyte levels. But, previous studies have demonstrated that although cough and cold alter several other salivary contents like expelled nucleic acid, glucose, protein, and digestive enzyme (e.g., amylase) levels, it has little implications on the secreted electrolytes (Farzan 1990). Therefore, we can assume that our method will also be suitable for those patients. Another drawback of this study is that due to ethical issues we could not measure electrolyte levels in other biological fluids like sweat, or urine. Theoretically, there is no apprehension in measuring electrolyte levels of other biological fluids, but experimentally it needs to be checked. A reference range of $\mathrm{Na}^{+}$and $\mathrm{K}^{+}$has to be determined for each of the biological fluids for the inclusion of this technique in regular clinical practices. A more comprehensive large scale clinical study will follow this work to answer the clinical issues in details. 


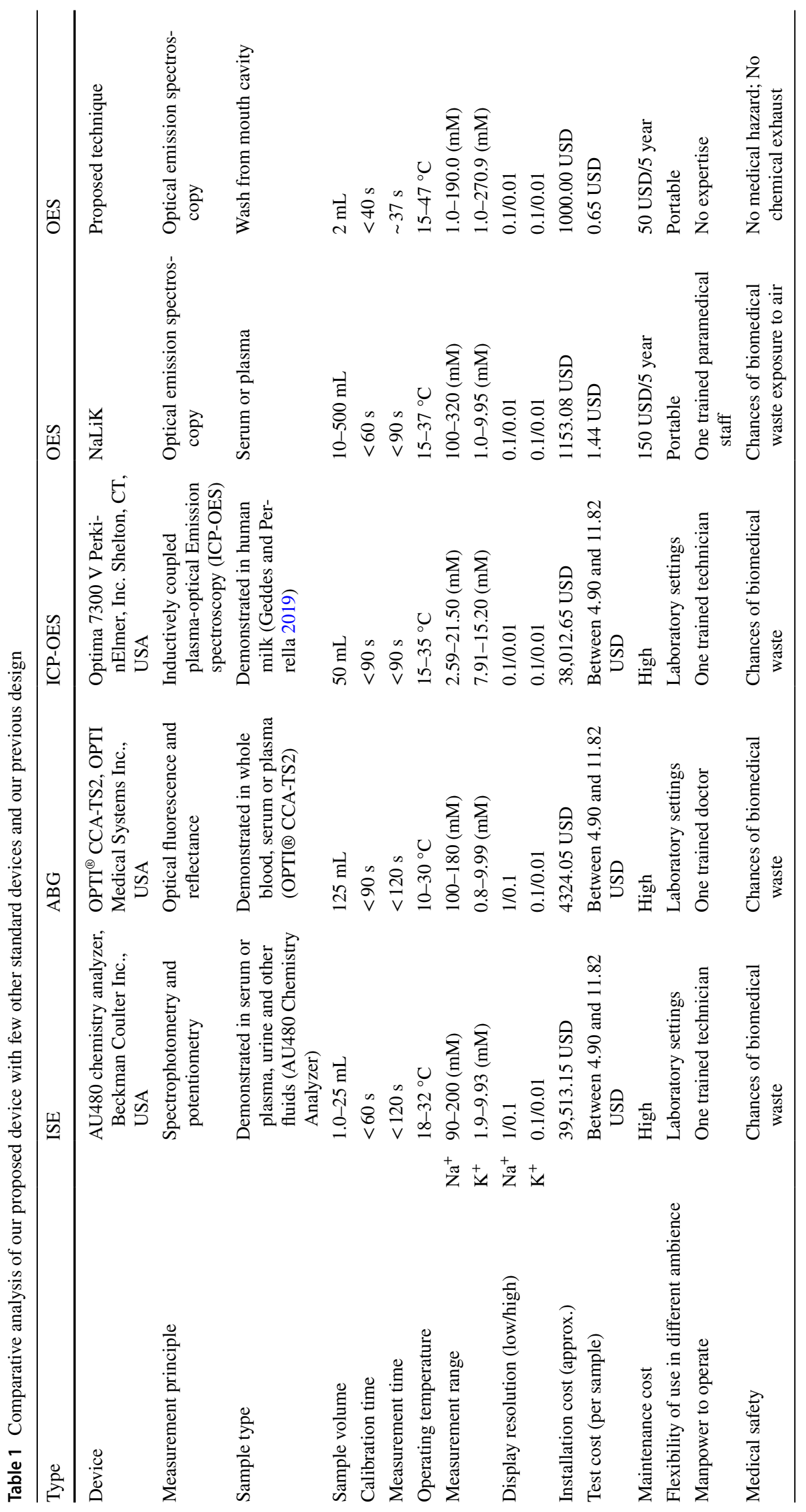




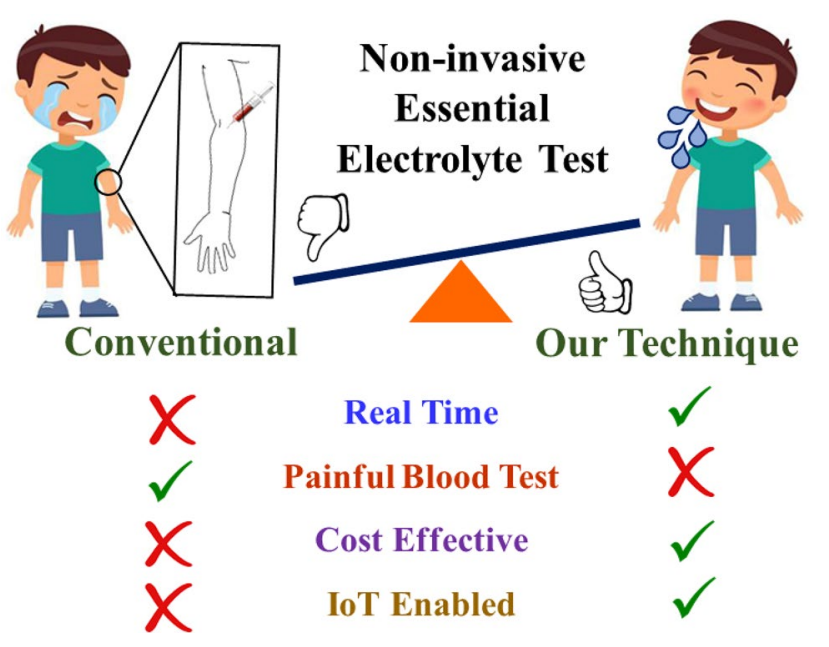

Fig. 7 Advantages of the developed techniques

\section{Conclusion}

In the present work, we have developed a non-invasive technique for the detection of essential electrolyte levels in human subjects using optical emission spectroscopy from samples collected from the wash of the mouth cavity. We have also developed a user interfacing software in the LabView platform to acquire the optical signals and to calculate the electrolyte concentration instantaneously. The technique has been validated through a small-scale clinical trial on 30 healthy volunteers. The obtained values of $\mathrm{Na}^{+}$and $\mathrm{K}^{+}$concentrations are found to be consistent with that of the reported values. The advantages of the developed system over the conventional blood sample-based technique include real-time analysis of the data, painless, needle-free sample collection and IoT-enabled e-healthcare strategy as remarkable as shown in Fig. 7. To our understanding, the co-morbidity of CKD and elderly patients due to frequent hospital visits and immune-compromised conditions can be managed in the COVID-19 pandemic situation through the developed device. A large-scale clinical study will be followed to address the clinical issues in further detail. Since our study was restricted with limited ethical permission, we are aiming to do further studies using other biological fluids, such as urine and sweat samples.

Acknowledgement SKP acknowledges the Indian National Academy of Engineering (INAE) and Science and Engineering Research Board (SERB), Department of Science and Technology (DST), Government of India for Abdul Kalam Technology Innovation National Fellowship (INAE/121/AKF).

Author contributions NB developed the instrument, and was involved in the planning of the study; coordinated data collection carried out the primary analyses, and drafted an initial manuscript. SS developed the software and revised the manuscript. AH was involved in the design and development of the instrument and reviewed the manuscript. AA analyzed the data, interpreted it, and revised the manuscript. RG made a substantial contribution to data acquisition and interpretation. DS helped in the design and development of the device. SKT, AKM, and PM planned the study interpreted the data, and reviewed the manuscript. SKP conceptualized and planned the study, interpreted the data, and revised the manuscript.

Data availability The datasets generated for this study are available on legitimate request to the corresponding author.

\section{Compliance with ethical standards}

Conflicts of interest There are no conflicts to declare.

\section{References}

Ajaimy M, Melamed ML (2020) COVID-19 in patients with kidney disease. Am Soc Nephrol 15(8):1087-1089

AU480 Chemistry Analyzer, https://www.beckmancoulter.com/produ cts/chemistry/au 480 .

Behl T et al (2020) The dual impact of ACE2 in COVID-19 and ironical actions in geriatrics and pediatrics with possible therapeutic solutions. Life Sci 15:118075

Chiles V et al (1996) Principles of engineering manufacture, 3rd edn. Butterworth-Heinemann. ISBN 0080539610

Declaration H (2014) World Medical Association Declaration of Helsinki. Retrieved Jan, 2014. 22:2014.

Delanghe JR (2019) Management of electrolyte disorders: also the method matters! Acta Clin Belg 74(1):2-6

Delves H (1987) Atomic absorption spectroscopy in clinical analysis. Ann Clin Biochem 24(6):529-551

Diwakar PK, Kulkarni P (2012) Measurement of elemental concentration of aerosols using spark emission spectroscopy. J Anal At Spectrom 27(7):1101-1109

Durst RA (1978) Sources of error in ion-selective electrode potentiometry in ion-selective electrodes in analytical chemistry. Springer, New York, pp 311-338

Farzan S (1990) Cough and sputum production, in Clinical Methods: the History, Physical, and Laboratory Examinations. 3rd edn. Butterworths.

Fraser SD, Blakeman T (2016) Chronic kidney disease: identification and management in primary care. Pragmatic Observ Res 7:21

Garcia RP et al (1992) Serum analysis for potassium ions using a fibre optic sensor. Clin Chim Acta 207(1-2):31-40

Geddes D, Perrella S (2019) Nutrients 11:802

Giavarina D (2019) Blood biochemistry: measuring major plasma electrolytes critical care nephrology. Elsevier, Philadelphia, pp 320-322

Halder A et al (2019) NaLiK, an self-developed device for rapid, reliable and simultaneous assessment of sodium, lithium and potassium for management of fluid balance and bipolar disorder in human subjects. J Anal At Spectrom 34(9):1875-1881

Hamming I et al (2007) The emerging role of ACE2 in physiology and disease. J Pathol 212(1):1-11

Hnatiuc B et al (2011) Spectroscopic diagnostic of transient plasma produced by a spark plug. Rom J Phys 56(SUPPL):109-113

Horiba (2017) Monitoring potassium levels in human saliva for mitigating periodic paralysis. Laqua twin 2403

Johns Hopkins University (JHU) (2021) COVID-19 Dashboard by the Center for Systems Science and Engineering (CSSE) at Johns Hopkins University (JHU). Johns Hopkins University (JHU). https ://coronavirus.jhu.edu/map.html 
Labat C et al (2018) Differential associations for Salivary Sodium, Potassium, Calcium, and Phosphate levels with Carotid Intima media thickness, heart rate, and arterial stiffness. Dis Markers 2018:3152146

Lee J et al (2019) An integrated ion-selective optode sensor cartridge for directly detecting electrolyte ions in blood plasma without pretreatment to adjust $\mathrm{pH}$. Sens Actua B Chem 280:256-262

Mizuiri S, Ohashi Y (2015) ACE and ACE2 in kidney disease. World J Nephrol 4(1):74

OPTI® CCA-TS2 Blood Gas and Electrolyte Analyzer, https://www. optimedical.com/products-services/opti-CCATS2.html.

Pinheiro DS et al (2019) The combination of ACE I/D and ACE2 G8790A polymorphisms revels susceptibility to hypertension: a genetic association study in Brazilian patients. PLoS ONE 14(8): $\mathrm{e} 0221248$

Rieder MJ et al (1999) Sequence variation in the human angiotensin converting enzyme. Nat Genet 22(1):59-62

Rose DP et al (2014) Adhesive RFID sensor patch for monitoring of sweat electrolytes. IEEE Trans Biomed Eng 62(6):1457-1465

Schneyer LH, Young JA, Schneyer CA (1972) Salivary secretion of electrolytes. Physiol Rev 52(3):720-777

Sher E et al (1992) A corona spark plug system for spark-ignition engines. SAE Technical Paper.

Shrivastava A, Gupta VB (2011) Methods for the determination of limit of detection and limit of quantitation of the analytical methods. Chron Young Sci 2(1):21

Singh S et al (2014) Spark spectrometry of toxic smokes: towards a portable, inexpensive, and high-resolution environment monitoring instrument. Clean Technol Environ Policy 16(8):1703-1712
Slanger T, Osterbrock D (2000) Investigations of potassium, lithium, and sodium emission in the nightglow and $\mathrm{OH}$ cross calibration. J Geophys Res 105(D1):1425-1429

Soler MJ et al (2013) Angiotensin-converting enzyme 2 activity in patients with chronic kidney disease. Nephrol Dial Transplant 28(11):2687-2697

Staack D et al (2008) Nanoscale corona discharge in liquids, enabling nanosecond optical emission spectroscopy. Angew Chem 120(42):8140-8144

Wakahara S et al (2007) Synergistic expression of angiotensin-converting enzyme (ACE) and ACE2 in human renal tissue and confounding effects of hypertension on the ACE to ACE2 ratio. Endocrinology 148(5):2453-2457

Wankasi MM et al (2019) Glucose and electrolytes concentrations in blood and saliva samples amongst diabetics. J Diabet Mellit 9(2):39-49

White AG et al (1955) Physiological and pharmacological regulation of human salivary electrolyte concentrations; with a discussion of electrolyte concentrations of some other exocrine secretions. J Clin Investig 34(2):246-255

Zungu LI, Sengane M, Setswe KG (2008) Knowledge and experiences of needle prick injuries (NPI) among nursing students at a. South African Family Pract 50(5):48-48

Publisher's Note Springer Nature remains neutral with regard to jurisdictional claims in published maps and institutional affiliations. 